\title{
DYNAMICS COEFFICIENT FOR TWO-PHASE SOIL MODEL
}

\author{
BOGUMIE WRANA \\ Cracow University of Technology, Faculty of Civil Engineering, Kraków, Poland, e-mail: bwrana@interia.pl
}

\begin{abstract}
The paper investigates a description of energy dissipation within saturated soils-diffusion of pore-water. Soils are assumed to be two-phase poro-elastic materials, the grain skeleton of which exhibits no irreversible behavior or structural hysteretic damping. Description of motion and deformation of soil is introduced as a system of equations consisting of governing dynamic consolidation equations based on Biot theory. Selected constitutive and kinematic relations for small strains and rotation are used. This paper derives a closed form of analytical solution that characterizes the energy dissipation during steady-state vibrations of nearly and fully saturated poro-elastic columns. Moreover, the paper examines the influence of various physical factors on the fundamental period, maximum amplitude and the fraction of critical damping of the Biot column. Also the so-called dynamic coefficient which shows amplification or attenuation of dynamic response is considered.
\end{abstract}

Key words: dynamic consolidation, Biot theory, dynamics, two-phase soil model

\section{INTRODUCTION}

Soil is a heterogeneous material consisting of mineral or organic grains or man-made with different sizes and pores occupied by fluid (mainly water) or gas (mainly air). Soil is, therefore, a three-phase material consisting of a solid phase (structure built of particles), liquid phase and gas phase. There are three approaches to the accuracy of soil models: singlephase model as a mixture of three-phase, two-phase model consisting of a skeleton and the liquid phase in the pores and three-phase model which takes into account the interaction of the three phases.

The work of Maurice Anthony Biot in 1956 is assumed to be the beginning of the theory of soil porous media development. Biot considered the wave propagation in a porous soil medium, using generalized Lagrangian displacement. He decided to separate the soil skeleton displacement $\mathbf{u}^{s}$ with fluid displacement $\mathbf{u}^{f}$ and considered kinetic energy and dissipation energy on the relative motion of fluid to the skeleton.

The paper investigates a description of energy dissipation within two-phase saturated soils-diffusion of pore-water.

\section{DYNAMIC LOADS}

The loads acting on the soil can be static, unchanging over time and/or dynamic varying over time.
This paper relates to dynamic loads which extend (relative to the static load) to identify the inertial forces dependent on the density and the soil acceleration.

Dynamic load (Fig. 1) acting on the soil can be divided, on the basis of the force dynamic characteristics, to the following groups:

- periodic (in soil dynamics often called cyclic), e.g., from a vibrating compaction or vibration of rotating machines installed on foundations, such as the turbines, fans, screens, etc.;

- impact or impulse acts directly on the soil or through the foundation on which shock devices, such as hammers, presses, etc., are mounted;

- seismic derived from earthquakes and para-seismic, derived from human activities, such as explosions in the mines or moving vehicles.

These loads are of different amplitude-frequency characteristics, and may be stationary with a constant spectrum in time or non-stationary with the spectrum changing over time.

In Fig. 1, a yellow area indicates dynamic loads acting for several minutes, during which there may occur two radically different phenomena in the soil. In the case of cohesive soils (e.g., silt or clay), where the permeability coefficient is small, dynamic analysis is reduced to a problem without drainage, which significantly simplifies the equations of motion. In the case of cohesionless soil (e.g., sand or gravel), where the permeability coefficient is large, dynamic analysis includes the problem of soil liquefaction (especially in the case of semi-compacted sand). 


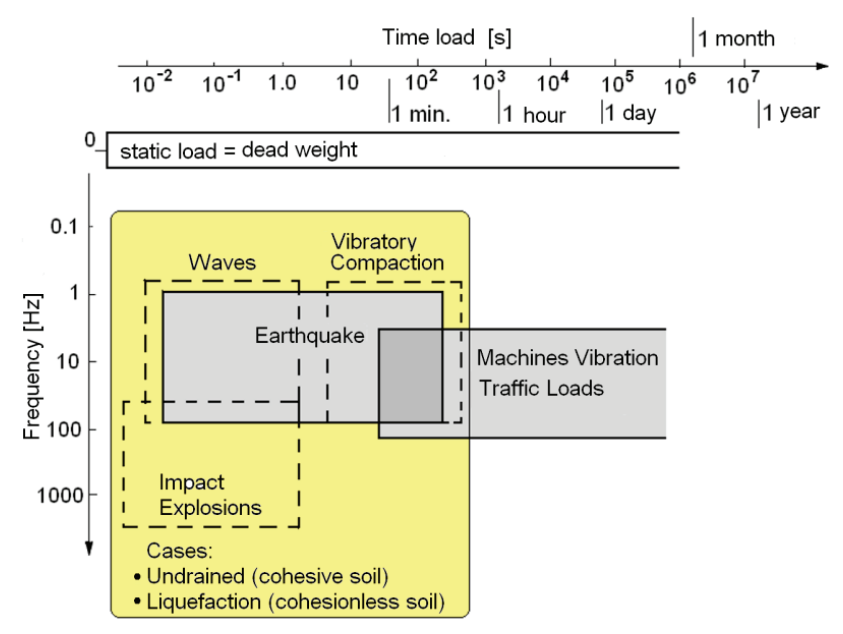

Fig. 1. Load types acting on the soil area

In the case of dynamic load transmitted to the soil, the decisive role as the constitutive relation between strain and stress is played by the soil model.

\section{BIOT THEORY}

Biot theory describes the wave propagation in saturated porous media as a soil skeleton fully saturated with fluid. Biot [3], [4] assumed that the motion of porous media on the micro level can be described by continuum mechanics of material. He used Lagrange postulate and Hamilton's principle to derive the equations of wave propagation.

The basic assumption of the equations of motion is the homogenization theory (the literature related to the theory is very extensive), for example, the volume averaging method used to describe the micro- and macroscopic relation.

Many books describe Biot theory of wave propagation and deformation theory of the porous medium, inter alia: Rice and Cleary [15]; Johnson [11]; Bourbié at al. [5]; Cristescu [10]; Zimmermann [22]; Allard [1]; Corapcioglu and Tuncay [8]; Mavko et al. [14]; Kubik et al. [12]; Wang [17]; Cederbaum, Li and Schulgasser [7]; Santamarina et al. [16]; Carcione [6]; Coussy [9].

The main assumptions of Biot theory are as follows:

1. In the porous medium there is a relationship between the current and reference state. Displacement, velocity and deformations of particles are small. Constitutive equations, dissipation forces and inertia forces are linear. Thus, the strain energy, dissipation potential and kinetic energy are a square form of variables.

2. Principles of continuum mechanics can be applied to measure the macroscopic value. The size of averaged macroscopic elementary volume is based on microscopic structure.

3. Compared to the macroscopic dimensions of the elementary volume, the wavelength is large. This volume sufficiently and accurately determines properties such as porosity, permeability and modulus of elasticity.

4. The isothermal state is under consideration.

5. The hydrostatic stress is assumed to fill the pores with viscous fluid.

6. The liquid phase is a continuum. The skeleton is a solid phase with discrete discontinuous areas.

\section{EQUATION OF MOTION IN LOW FREQUENCY RANGE}

The formulation of porous media dynamics is based on the kinetic energy equation, dissipation function and it leads to the equations of motion using Lagrange's postulate.

For two-phase soil model the equation of motion is as follows (Wrana [18])

$$
\frac{\partial \sigma_{i j}}{\partial x_{j}}=\left(\rho_{11}+\rho_{12}\right) \frac{\partial^{2} u_{i}^{m}}{\partial t^{2}}+\left(\rho_{12}+\rho_{22}\right) \frac{\partial^{2} u_{i}^{f}}{\partial t^{2}}
$$

or in the matrix form

$$
\operatorname{div} \boldsymbol{\Sigma}=\left(\rho_{11}+\rho_{12}\right) \frac{\partial^{2}}{\partial t^{2}} \mathbf{u}^{m}+\left(\rho_{12}+\rho_{22}\right) \frac{\partial^{2}}{\partial t^{2}} \mathbf{u}^{f} .
$$

Assuming the relative displacement of skeleton to fluid

$$
w_{i}=n\left(u_{i}^{f}-u_{i}^{m}\right)
$$

or in matrix form

$$
\mathbf{w}=n\left(\mathbf{u}^{f}-\mathbf{u}^{m}\right) .
$$

\section{PROBLEM FORMULATION FOR 1D PROBLEM}

In general, soils are three-phase media that consist of solid, fluid and gas constituents with masses $M_{s}$, 
$M_{f}, M_{g}$ and volumes $V_{s}, V_{f}, V_{g}$, respectively. The volume $V_{p}$ of pores and the total volume $V_{t}$ of the medium are

$$
V_{p}=V_{f}+V_{g}, \quad V_{t}=V_{s}+V_{f}+V_{g} .
$$

In the paper, the porosity $n=V_{p} / V_{t}$ and the degree of saturation $S=V_{f} / V_{p}$ are considered. Therefore, only nearly saturated soils are considered for which $S$ is between 0.95 to 1.0. The gas is dissolved in the fluid, there is only one homogeneous fluid phase. The unit mass of the solid $\rho_{s}=\frac{M_{s}}{V_{s}}$ and of the fluid $\rho_{f}=\frac{M_{f}}{V_{f}}$ is practically independent of the degree of saturation $S$ and is equal to the unit mass of water $\rho_{w}=\frac{1 g}{\mathrm{~cm}^{3}}$. The specific gravity of the solid is $G_{s}=\frac{\rho_{s}}{\rho_{f}}$.

One-dimensional case is considered (Bardet [2]). The terms $u$ and $v$ denote solid and fluid displacements, respectively. The linear strain-displacement relationship is $\varepsilon=\frac{\partial u}{\partial z}$. The change of effective stress resulting from $\varepsilon$ is

$$
\Delta \sigma^{\prime}=D \varepsilon
$$

where the parameter of solid material $D=\frac{E(1-v)}{(1-2 v)(1+v)}$.

The change in the fluid pressure is

$$
\Delta p=\frac{D_{f} \partial v}{\partial z}
$$

where $D_{f}$ - bulk modulus of the air-fluid mixture depends on the degree of saturation $S$.

Assuming that particles are incompressible and water flow through the solid grains obeys Darcy's law, the governing equations are

$$
\begin{aligned}
& (D+Q) u_{, z z}+D_{f} v_{, z z}=(1-n) \rho_{s} u_{, t t}+n \rho_{f} v_{, t t}, \\
& Q u_{, z z}+D_{f} v_{, z z}=\rho_{f}\left[v_{, t t}+\frac{n g}{k}\left(v_{, t}-u_{, t}\right)\right],
\end{aligned}
$$

where

$$
\begin{aligned}
& Q=\frac{1-n}{n} D_{f}, \\
& u(z, t)-\text { displacement of skeleton, } \\
& v(z, t)-\text { displacement of water, } \\
& k \quad-\text { permeability coefficient, } \\
& g \quad-\text { acceleration due to gravity. }
\end{aligned}
$$

After introducing new constants:

$\chi=\frac{D}{D_{f}}-$ solid bulk modulus to air-fluid mixture modulus,

$$
\beta=\frac{1}{\left[n+(1-n) G_{s}\right]}-\text { solid density to air-fluid den- }
$$
sity,

$c_{f}=\sqrt{\frac{D_{f}}{\rho_{f}}}-$ air-fluid wave longitudinal velocity,

$w=n(v-u)-$ relative displacement fluid to skeleton.

Equations (6) become

$$
\begin{aligned}
& \frac{c_{f}^{2}}{n}\left[(1+n \chi) u_{, z z}+w_{, z z}\right]=\frac{1}{\beta} u_{, t t}+w_{, t t}, \\
& \frac{c_{f}^{2}}{n}\left(u_{, z z}+w_{, z z}\right)=u_{, t t}+\frac{1}{n} w_{, t t}+\frac{g}{k} w_{, t} .
\end{aligned}
$$

Equation (7) takes into account the viscous damping between the grains and the water in the pores by component $\frac{g}{k} w_{, t}$.

\section{STEADY STATE RESPONSE OF THE BIOT COLUMN}

The Biot column is considered. At the top $(z=0)$ free drained displacement is subjected to harmonic normal stress $\sigma_{0}$ with circular frequency $\omega$ and it is clamped and undrained at the base $(z=L)$ (see Fig. 2).

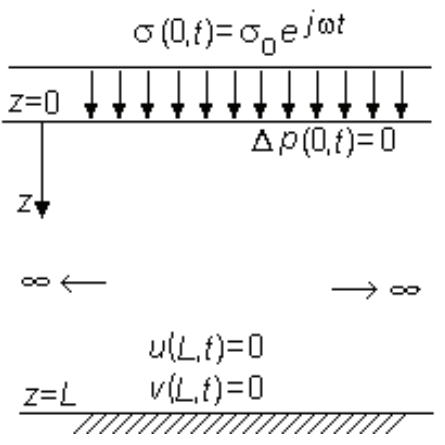

Fig. 2. Geometry and boundary conditions of the Biot column

According to separation rules of variables in space and time, the following is obtained 


$$
u(z, t)=U(z) e^{j \omega t} \quad \text { and } \quad w(z, t)=W(z) e^{j \omega t}
$$

where $j=\sqrt{-1}$, and solution in space as

$$
U(z)=A e^{\lambda z} \quad \text { and } \quad W(z)=B e^{\lambda z} .
$$

Introducing (8) to (6) and using new complex constant $\psi=\frac{c_{f} \lambda}{\omega}$, two linear algebraic equations with two unknowns $A$ and $B$ are obtained

$$
\begin{aligned}
& A\left[\psi^{2}\left(\chi+\frac{1}{n}\right)+\frac{1}{\beta}\right]+B\left(\frac{\psi^{2}}{n}+1\right)=0, \\
& A\left(\frac{\psi^{2}}{n}+1\right)+B\left(\frac{\psi^{2}}{n}+\frac{1}{n}-j \frac{g}{k \omega}\right)=0 .
\end{aligned}
$$

Dynamic factor $\gamma$ is introduced as

$$
\gamma=\frac{20 \log _{10} U_{d y n}}{U_{\text {stat }}}
$$

where $20 \log _{10}$ - coefficient for unit decibels.

The nontrivial solution is obtained from characteristic equation of (10)

$$
\begin{gathered}
U(z)=A_{1} e^{\lambda_{1} z}+A_{2} e^{\lambda_{2} z}+A_{3} e^{-\lambda_{1} z}+A_{4} e^{-\lambda_{2} z}, \\
W(z)=K_{1} A_{1} e^{\lambda_{1} z}+K_{2} A_{2} e^{\lambda_{2} z}+K_{1} A_{3} e^{-\lambda_{1} z}+K_{2} A_{4} e^{-\lambda_{2} z},
\end{gathered}
$$

where $K_{1}, K_{2}$ - from equation (10), and $A_{1}, A_{2}, A_{3}$, $A_{4}$ - from boundary conditions of (12).

After fulfilling the four boundary conditions we obtain the solution for dynamic coefficient $\gamma$ as
With reference to the undrained case, the calculation frequency is defined as

$$
\Omega=\frac{\omega L}{c_{f}}[\mathrm{rad}] .
$$

In the example a semi-compacted sand layer with thickness $L=10 \mathrm{~m}$ was considered. The parameters used in the calculation are shown in Table 1. Figures 3 and 4 show the answer to Biot column $U(z)$ at the depth $z=0$. Figure 3 shows the dynamic coefficient value for skeletal soil, depending on the relation of soil stiffness to liquid stiffness in frequency parameter $\Omega$. Figure $3 \mathrm{~b}$, c shows the areas of increasing $(\gamma>0)$ and damping $(\gamma<0)$ of skeleton displacement amplitudes $U(z)$.

Figure 3 shows the results of dynamic coefficient $\gamma$ and Fig. 4. shows the influence of permeability coefficient $k$ on displacement amplitude of $u$-skeleton, $v$-water and $p$ - pore water pressure.

Table 1. Parameter

\begin{tabular}{|c|c|c|}
\hline$L[\mathrm{~m}]$ & $\sigma_{0}[\mathrm{kPa}]$ & $\rho_{s}\left[\mathrm{t} / \mathrm{m}^{3}\right]$ \\
\hline 10 & 100 & 2.65 \\
\hline
\end{tabular}

\section{CONCLUSION}

The harmonic response of Biot column of fullysaturated poro-elastic soil was analytically solved. A parametric study revealed the influence of soil and fluid rigidity on frequency.

$$
\gamma=\frac{20 \log _{10} U_{d y n}}{U_{\text {stat }}}=20 \log _{10}\left\{\frac{1}{K_{2}-K_{1}}\left[\frac{\left(K_{2}+1\right) \sinh \left[\lambda_{1}(z-L)\right]}{\lambda_{1} L \cosh \left[\lambda_{1} L\right]}-\frac{\left(K_{2}+1\right) \sinh \left[\lambda_{2}(z-L)\right]}{\lambda_{2} L \cosh \left[\lambda_{2} L\right]}\right]\right\} .
$$

For $\gamma>0$, the amplitude of vibration is increased compared to the static displacement, which is analogous to a dynamic system with one degree of freedom as the resonance area. For $\gamma<0$ the amplitude vibration is less than that of a solution for the static, outside the resonance area.

\section{EXAMPLE}

Various physical factors were considered in the paper. The problem of resonance in skeleton depends on:

$D / D_{f}-$ skeleton stiffness/fluid (water) stiffness, and

$$
c_{0}=c_{f} \sqrt{\beta\left(\frac{D}{D_{f}}-\frac{1}{n}\right)}-\text { undrained wave velocity. }
$$

Results presented in Fig. 3 indicate that:

- Maximum value of dynamic coefficient $\gamma$ is for $\Omega$ $=\pi / 2,3 / 2 \pi, 5 / 2 \pi$, etc. that is, the values of resonance (see Fig. 3a).

- Values of resonance amplitude decrease with the increase of $\Omega$. The largest value of the frequency amplitude is for $\Omega=\pi / 2$, as shown in Fig. 3b,c and increases with the axis ratio $D / D_{f}$,

- In areas between $\pi / 2,3 / 2 \pi, 5 / 2 \pi$, etc. the skeleton displacement amplitude is reduced. The lowest values are at frequency parameter $\Omega=\pi$, $2 \pi, 3 \pi$, etc.

- According to the Terzaghi equation for maximum values of skeleton displacement amplitude, the liquid amplitudes are minimum values. 


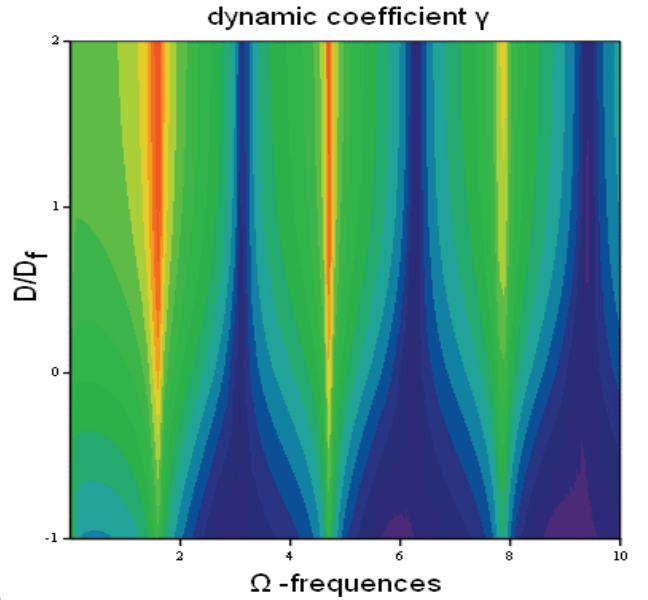

(a)

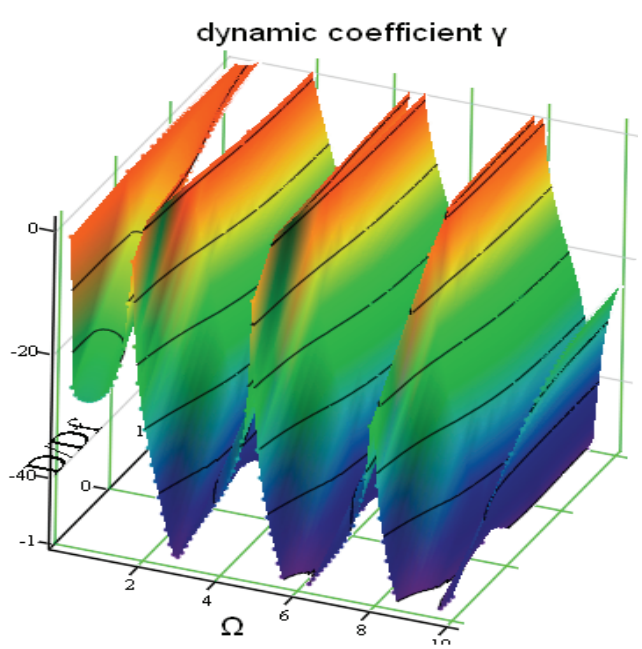

(b)

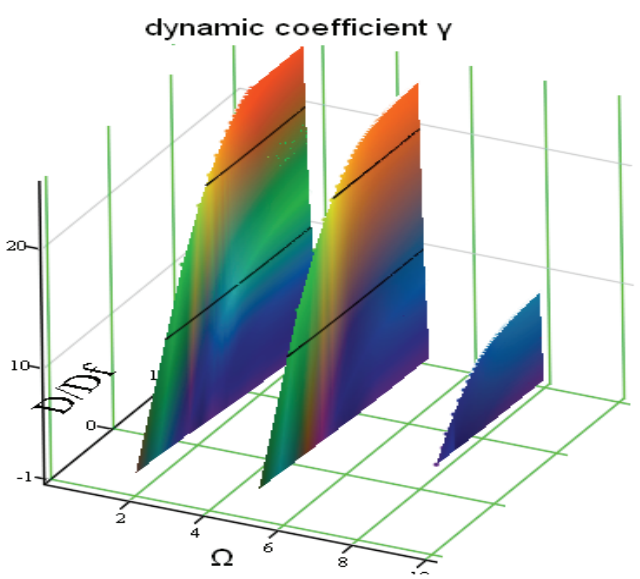

Fig. 3. Value of dynamic coefficient $\gamma$ depends on $D / D_{f}-$ skeleton stiffness/water stiffness and $\Omega$.

(a) dynamic coefficient $\gamma$, (b) $\gamma<0$ - damping area,

(c) $\gamma>0-$ resonance area

Results presented in Fig. 4 indicate that the permeability coefficient $k$ plays an important role in the dynamic behavior. Figure 4 shows relation of skeleton and water displacement and pore pressure to permeability coefficient $k$ and calculation frequency $\Omega$. One can find the result for sand with three different values of $k: k=0.0001 \mathrm{~m} / \mathrm{s}(\mathrm{FSa}), k=0.001 \mathrm{~m} / \mathrm{s}(\mathrm{MSa})$ and $k=0.01 \mathrm{~m} / \mathrm{s}(\mathrm{CSa})$.

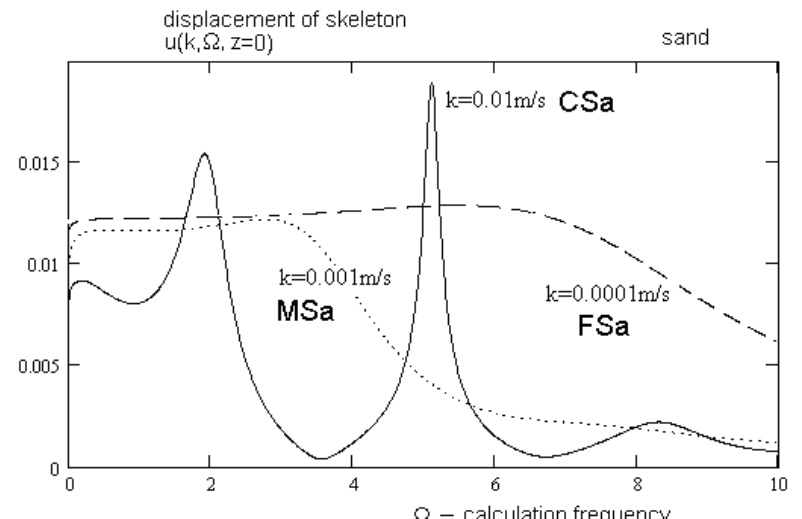

a)
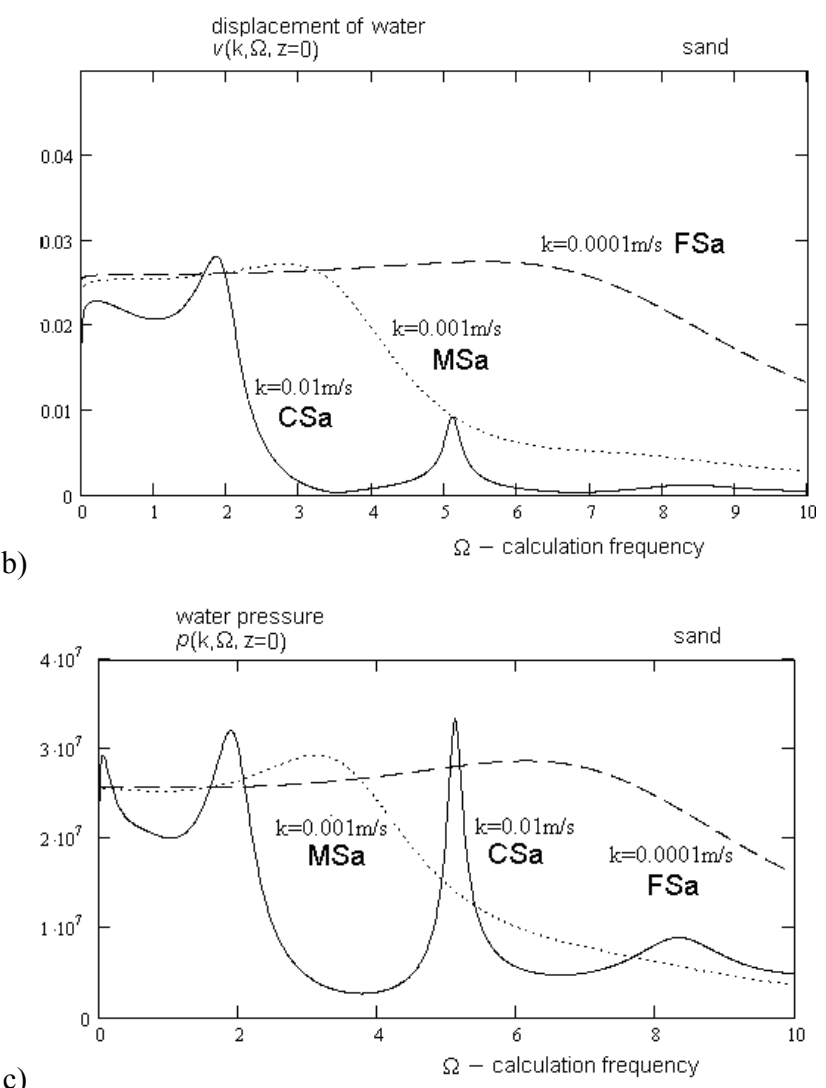

Fig. 4. Influence of permeability coefficient $k$ on displacement amplitude of (a) $u$, (b) $v$, and (c) $p$

\section{REFERENCES}

[1] Allard J.F., Propagation of Sound in Porous Media, Elsevier Science Publ., 1993.

[2] BARdet J.P., The Damping of Saturated Poroelastic Soils during Steady State Vibration, Applied Mathematics and Computation, 67, 1995, 3-31.

[3] Biot M.A., Theory of Propagation of Elastic Waves in a Fluidsaturated Porous Solid. I. Low-frequency range, J. Acoust. Soc. Am., 28, 1956a, 168-178. 
[4] BIOT M.A., Theory of Propagation of Elastic Waves in a Fluidsaturated Porous Solid. II. High-frequency Range, J. Acoust. Soc. Am., 28, 1956b, 179-191.

[5] Bourbié T., Coussy, O., Zinszner, B., Acoustics of Porous Media, Editions Technip., 1987.

[6] CARcione J.M., Wave Fields in Real Media: Wave Propagation in Anisotropic, Elastic and Porous Media, Elsevier Science Ltd., 2001.

[7] Cederbaum G., Li L., Schulgasser K., Poroelastic Structures, Elsevier Science, 2000.

[8] Corapcioglu M.Y., Tuncay K., Propagation of Waves in Porous Media, [in:] Corapcioglu M.Y. (ed.), Advances in Porous Media, 3, Elsevier Science Pub. Co. Inc., 1996, 361-440.

[9] Coussy O., Poromechanics, Wiley, Chichester, UK, 2004.

[10] Cristescu N., Rock Rheology, Kluwer Academic Publ., 1986.

[11] Johnson D.L., Recent Developments in the Acoustic Properties of Porous Media, [in:] D. Sette (ed.), Frontiers in Physical Acoustics, Proceedings of the International School of Physics "Enrico Fermi", Course 93, 1986, 255-290.

[12] KubiK J., Cieszko M., Kaczmarek M., Basic Dynamics of Saturated Porous Media, Biblioteka Mechaniki Stosowanej, Seria A. Monografie (in Polish), 2000.

[13] Lewis R.W., Schrefler B.A., The Finite Element Method in the Static and Dynamic Deformation and Consolidation of Poruos Media, John Willey \& Sons, 1998.
[14] Mavko G., MukerJi, T., Dvorkin J., The Rock Physics Handbook: Tools for Seismic Analysis in Porous Media, Cambridge Univ. Press, 1998.

[15] Rice J.R., Cleary M.P., Some Basic Stress Diffusion Solutions for Fluid Saturated Elastic Porous Media with Compressible Coefficients, Rev. Geophys., 14, 1976, $227-$ 241.

[16] Santamarina J.C., Klein K.A., FAm M.A., Soils and Waves: Particulate Materials Behavior, Characterization and Process Monitoring, John Wiley \& Sons, 2001.

[17] Wang H.F., Theory of Linear Poroelasticity, with Applications to Geomechanics and Hydrogeology, Princeton University Press, 2000.

[18] Wrana B., Computational Models of Soil Dynamics, Cracow University of Technology (in Polish), 2012.

[19] Wrana B., PietrZaK N., Influence of Inertia Forces on Soil Settlement under Harmonic Loading, Studia Geotechnica et Mechanica, No. 1, 2013, 245-258.

[20] Zamman M., Gioda G., Booker J., Modelling in Geomechanics, John Wiley \& Sons, 2000.

[21] Zienkiewicz O.C., Chan A.H.C., Pastor M., Schrefler B.A., Shiomi T., Computational Geomechanics with Special Reference to Earthquake Engineering, John Willey \& Sons, 2000.

[22] Zimmerman R.W., Compressibility of Sandstones, Elsevier Science Publ., 1991. 